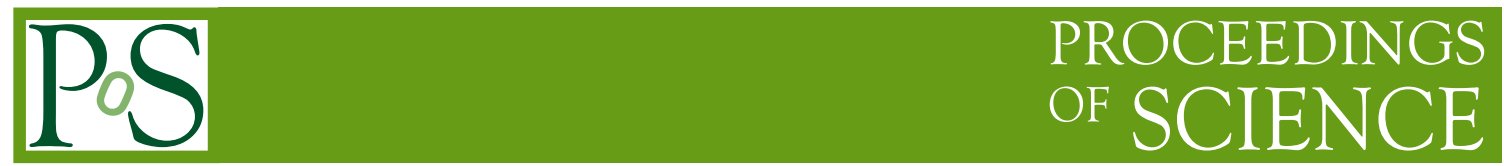

\title{
Upsilon suppression in heavy-ion collisions at LHC energies
}

\section{Felix Nendzig and Georg Wolschin* \\ Univ. Heidelberg ITP \\ E-mail: wolschin@uni-hd.de}

\begin{abstract}
We suggest that the combined effect of screening, gluon-induced dissociation, collisional damping, and reduced feed-down explains most of the sequential suppression of $\Upsilon(n S)$ states that has been observed in $\mathrm{PbPb}$ relative to $p p$ collisions at $\sqrt{s_{N N}}=2.76 \mathrm{TeV}$. The suppression is thus a clear, albeit indirect, indication for the presence of a QGP. The $\Upsilon(1 S)$ ground state suppression is essentially due to reduced feed-down, collisional damping and gluodissociation, whereas screening prevails for the suppression of the excited states.
\end{abstract}

Xth Quark Confinement and the Hadron Spectrum

$8 \ddot{E E} 12$ October 2012

TUM Campus Garching, Munich, Germany

${ }^{*}$ Speaker. 


\section{Introduction}

The suppression of quarkonium $(Q \bar{Q})$ states is one of the most promising probes for the properties of the quark-gluon plasma (QGP) that is generated in heavy-ion collisions at highly relativistic energies. In the QGP the confining potential is screened due to the interaction of the heavy $Q \bar{Q}$ with medium partons and hence, charmonium and bottomium states successively melt [1] at sufficiently high temperatures $T_{\text {diss }}$ beyond the critical value $T_{c} \approx 170 \mathrm{MeV}$.

However, additional processes such as gluon-induced dissociation, and collisional damping contribute to the suppression, and are effective in a temperature region where the $\Upsilon(n S)$ states and in particular, the $\Upsilon(1 S)$ ground state - have not yet melted due to screening.

Here we concentrate on such processes. It turns out that in particular for the $\Upsilon(1 S)$ ground state, bottomium dissociation is not just static screening, but mostly caused by other means whereas the dissociation of the excited states is essentially due to screening.

Charmonium suppression has been studied since 1986 in great detail both theoretically [2, 3, 4], and experimentally at energies reached at the CERN SPS, BNL RHIC [5], and the CERN LHC $[6,7]$. Bottomium suppression is expected to be a cleaner probe. The $\Upsilon(1 S)$ ground state with mass $9.46 \mathrm{GeV}$ is strongly bound. It melts as the last $Q \bar{Q}$ in the QGP (depending on the potential) only at about $4.10 T_{c}$ [8]. Even at LHC energies the number of $b \bar{b}$-pairs in the QGP remains small such that statistical recombination is unimportant.

$\Upsilon$ suppression in heavy-ion collisions has recently been observed for the first time both by the STAR experiment at RHIC [9], and by the CMS experiment at LHC [10, 11]. Preliminary CMS data from the 2011 run [12] have much better statistics such that the $\Upsilon(2 S)$ state can now be resolved individually in $\mathrm{PbPb}$ collisions at the LHC. In this work we suggest a three step model that considers the $\Upsilon(1 S, 2 S, 3 S)$ and $\chi_{b}(1 P, 2 P)$ states to obtain the suppression of the $\Upsilon(1 S, 2 S, 3 S)$ states at LHC energies, which is then compared to the experimental results. We successively calculate

1. the $b \bar{b}$ wave functions, and decay widths for the three processes Debye screening, collisional damping and gluodissociation [14]

2. the suppression of the five states considered here within the expanding and cooling fireball

3. the feed-down cascade, and the ensuing fraction of dimuon decays, $\Upsilon(n S) \rightarrow \mu^{+} \mu^{-}$.

Whereas gluodissociation below $T_{c}$ is not possible due to confinement, it does occur above $T_{c}$ where the color-octet state of a free quark and antiquark can propagate in the medium. Its significance increases substantially with the rising gluon density at LHC energies.

In the midrapidity range $|y|<2.4$ where the CMS measurement $[10,12,11]$ has been performed, the temperature and hence, the thermal gluon density is high, and causes a rapid dissociation in particular of the $\Upsilon(2 S)$ and $\Upsilon(3 S)$ states, but also of the $\Upsilon(1 S)$ ground state. At larger rapidities up to the beam value of $y_{\text {beam }}=7.99$ and correspondingly small scattering angles where the valence-quark density is high [15], nonthermal processes would be more important than in the midrapidity region that we are investigating here. 


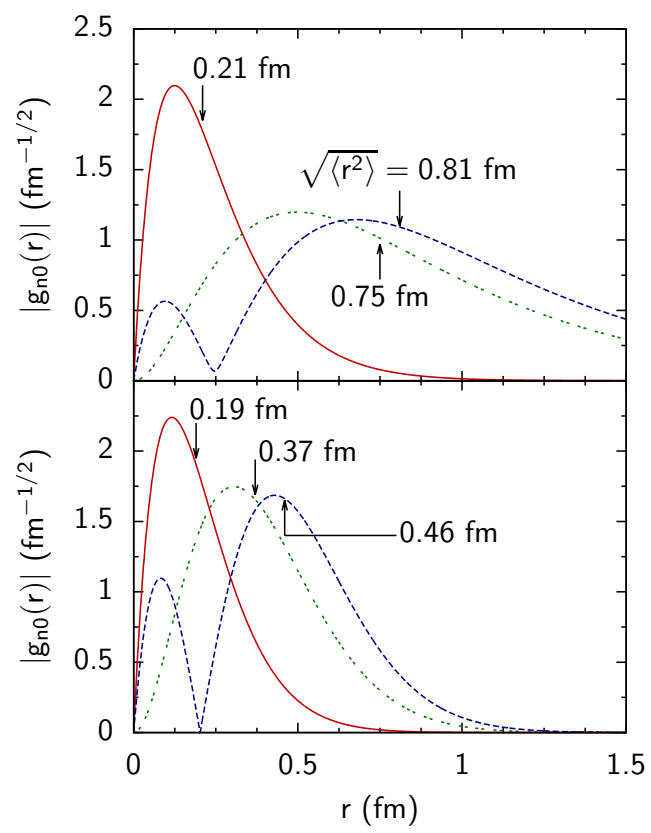

Figure 1: (Color online) Radial wave functions of the $\Upsilon(1 S), \chi_{b}(1 P)$ and $\Upsilon(2 S)$ states (solid, dotted, dashed curves, respectively) calculated in the complex, screened potential (2.1) for temperatures $T=0 \mathrm{MeV}$ (bottom) and $200 \mathrm{MeV}$ (top) with effective coupling constant $\alpha_{\text {eff }}=(4 / 3) \alpha_{s}^{s}=0.63$, and string tension $\sigma=0.192 \mathrm{GeV}^{2}$. While the rms radius $\sqrt{\left\langle r^{2}\right\rangle}$ of the $\Upsilon(1 S)$ ground state is almost insensitive to temperature changes, it varies substantially with temperature for the $\chi_{b}(1 P)$ and $\Upsilon(2 S)$ states. From [13].

\section{Bottomium wave functions and collisional damping}

Due to the small relative velocity $v \ll c$ of the bottom quarks in the bound state, $b \bar{b}$ may be properly described by the potential NonRelativistic QCD (pNRQCD) approach $[16,17,18]$. The relevant terms in the pNRQCD action for the $b \bar{b}$-pair can be calculated as in [19, 20, 21, 22].

This approach leads to a Schrödinger equation, with the coulombic, color-singlet potential $V=-C_{F} \alpha_{s}^{s} / r$, the strong coupling constant at the soft scale, $\alpha_{s}^{s}=\alpha_{s}\left(m_{b} \alpha_{s} / 2\right)=0.48$, and $N_{c}=3$, $C_{F}=\left(N_{c}^{2}-1\right) /\left(2 N_{c}\right)=4 / 3$. For the treatment of $b \bar{b}$ in the QGP it is, however, appropriate to make a calculation at finite temperature which yields for the short-range part of the potential, in the HTL approximation, a complex, screened, coulombic expression [23, 24].

The long range part is parameterized as in [25] so that the full singlet potential reads

$$
V\left(r, m_{D}\right)=\frac{\sigma}{m_{D}}\left(1-e^{-m_{D} r}\right)-\alpha_{e f f}\left(m_{D}+\frac{e^{-m_{D} r}}{r}\right)-i \alpha_{e f f} T \int_{0}^{\infty} \frac{d z 2 z}{\left(1+z^{2}\right)^{2}}\left(1-\frac{\sin \left(m_{D} r z\right)}{m_{D} r z}\right),
$$

with the Debye mass $m_{D}=T \sqrt{4 \pi \alpha_{s}^{T}\left(\frac{N_{c}}{3}+\frac{N_{f}}{6}\right)}, \alpha_{e f f}=4 \alpha_{s}^{s} / 3$, the number of flavors in the QGP $N_{f}=3$, and the strong coupling constant evaluated at the HTL energy $2 \pi T, \alpha_{s}^{T}=\alpha_{s}(2 \pi T) \leq 0.50$, respectively. The absolute values $\left|g_{n l}(r)\right|$ of the resulting $b \bar{b}$ wave functions are shown in Fig. 1.

The Schrödinger equation is now solved for every $b \bar{b}$ state with the potential (2.1) for $T \geq T_{c}$ up to the dissociation temperature $T_{\text {diss }}$ above which screening prevents bottomium formation and the 
Schrödinger equation has no bound states solutions. The dissociation temperatures with the above parameters are $T_{\text {diss }} \simeq 668,217$ and $206 \mathrm{MeV}$ for the $\Upsilon(1 S), \Upsilon(2 S)$ and $\chi_{b}(1 P)$, respectively: The higher excited states are already dissolved for $T \gtrsim T_{c}$. The imaginary part of the potential causes a decay width $\Gamma_{\text {damp }}$ which monotonically increases with temperature and accounts for collisional damping by the plasma particles.

\section{Gluodissociation in the medium}

Due to the high gluon density reached at LHC energies in the mid-rapidity region, gluodissociation is a major process besides screening and collisional damping that leads to a suppression of $\Upsilon$ 's at LHC. Hence we calculate the gluodissociation cross sections for the $\Upsilon(1 S)-\Upsilon(3 S)$, and $\chi_{b}(1 P), \chi_{b}(2 P)$ states for different lifetimes $t_{Q G P}$ of the QGP.

The leading-order dissociation cross section of the $b \bar{b}$ states through E1 absorption of a single gluon had been derived by Bhanot and Peskin (BP) [26]. From the pNRQCD approach the gluodissociation cross section may be derived from the dipole interaction term describing a singlet-octet transition of the $b \bar{b}$-pair via emission/absorption of an ultra soft gluon. From this starting point we can easily generalize the approach to include the effect of our modified potential (2.1) [27], and obtain for a bottomium state $(n l)$

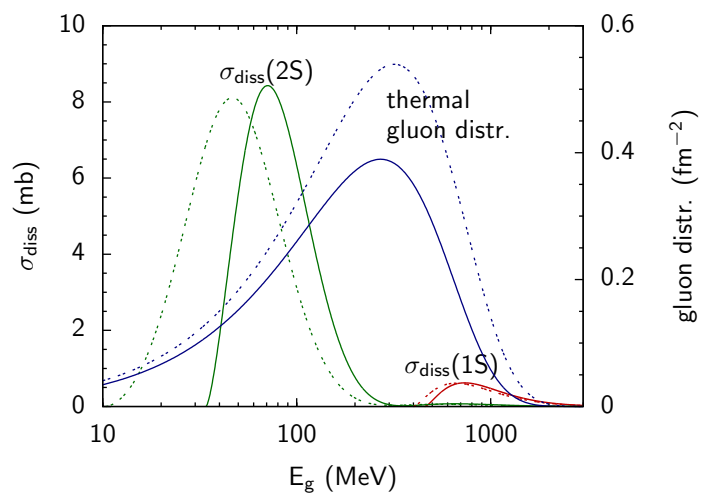

Figure 2: (Color online) Gluodissociation cross sections $\sigma_{\text {diss }}(n S)$ in mb (left scale) of the $\Upsilon(1 S)$ and $\Upsilon(2 S)$ states calculated using the screened complex potential for temperatures $T=170$ (solid curves) and $200 \mathrm{MeV}$ (dotted curves) as functions of the gluon energy $E_{g}$. The thermal gluon distribution (right scale; solid for $T=170 \mathrm{MeV}$, dotted for $200 \mathrm{MeV}$ ) is used to obtain the thermally averaged cross sections through integrations over the gluon momenta. From [13].

$$
\sigma_{d i s s, n l}\left(E_{g}\right)=\frac{2 \pi^{2} \alpha_{s}^{u} E_{g}}{(2 l+1) N_{c}^{2}} \sum_{m=-l}^{l} \sum_{l^{\prime}=0}^{\infty} \sum_{m^{\prime}=-l^{\prime}}^{l^{\prime}} \cdot \int_{0}^{\infty} d q\left|\left\langle n l m|\hat{\vec{r}}| q l^{\prime} m^{\prime}\right\rangle\right|^{2} \delta\left(E_{g}+E_{n l}-\frac{q^{2}}{m}\right)
$$

with the singlet and octet states $|n l m\rangle,\left|q l^{\prime} m^{\prime}\right\rangle$ and $\alpha_{s}^{u}=\alpha_{s}\left(m_{b} \alpha_{s}^{2} / 2\right) \simeq 0.59$. The radial wave function of the states $\left|q l^{\prime} m^{\prime}\right\rangle$ is derived from the octet Hamiltonian with the potential $V_{8}=+\alpha_{e f f} /(8 r)$.

We had originally derived the gluodissociation cross section in [14] independently from the pNRQCD formulation in an approach that was based on a straightforward extension of the BhanotPeskin formulation [26] to approximately account for the confining string contribution [27]. 
For vanishing string tension and the corresponding values of the binding energy $E_{n l}$, a pure Coulomb $1 S$ wave function, and a simplification in the octet wave function, our expression reduces to the result in [26]. Our full result for the $\Upsilon(1 S)$ gluodissociation cross section agrees with the result obtained independently by Brambilla et al. in their effective field theory approach $[21,22]$ in the limit discussed in [14].

To obtain the mean gluodissociation cross section, we average our calculated gluodissociation cross sections over the Bose-Einstein distribution function of gluons at temperature $T$, thus assuming that the medium is thermalized, although the heavy $b \bar{b}$ is not (see Fig. 2 for the gluon distribution):

$$
\Gamma_{\text {diss }, n l}=\frac{g_{d}}{2 \pi^{2}} \int_{0}^{\infty} \frac{d p_{g} p_{g}^{2} \sigma_{d i s s, n l}\left(E_{g}\right)}{e^{E_{g} / T}-1},
$$

where $g_{d}=16$ is the number of gluonic degrees of freedom. Taking $\Gamma_{\text {damp }}$ from the previous section together with the resulting width from gluodissociation yields the total decay width in the QGP, $\Gamma_{\text {tot }}=\Gamma_{\text {damp }}+\Gamma_{\text {diss }}$.

\section{Time evolution of the fireball and decay cascade}

The density distribution of the lead ions is modeled by a Woods-Saxon potential with radius $R=6.62 \mathrm{fm}$ and diffuseness $a=0.546 \mathrm{fm}$ [28]. The number $N_{b \bar{b}}$ of produced $b \bar{b}$-pairs at the point $(x, y)$ in the transverse plain and impact parameter $b$ is then proportional to the number of binary collisions $N_{c o l}$ and nuclear overlap $T_{A A}, N_{b \bar{b}}(b, x, y) \propto N_{\text {coll }}(b, x, y) \propto T_{A A}(b, x, y)$. The initial temperature is parametrized depending on the number of collisions, and Bjorken scaling is used for the time evolution [29]. We define a preliminary suppression factor $R_{A A}^{\text {prel }}$, which accounts only for the $b \bar{b}$ suppression due to the three processes Debye screening, collisional damping and gluodissociation,

$$
R_{A A}^{p r e l}=\frac{\int d^{2} b \int d x d y T_{A A}(b, x, y) e^{-\int_{t_{F}}^{\infty} d t \Gamma_{t o t}(b, t, x, y)}}{\int d^{2} b \int d x d y T_{A A}(b, x, y)}
$$

The numerator of eq. (4.1) is proportional to the number of $b \bar{b}$ bound states which have survived from their formation time $t_{F}$ until the fireball has cooled below the critical temperature $T_{c}$, where the decay width $\Gamma_{\text {tot }}$ is set to vanish.

Now that we have calculated the suppression during the evolution of the fireball we have to consider the feed-down of the remaining $b \bar{b}$ population to calculate the fraction of decays into dimuon pairs, $\Upsilon(n S) \rightarrow \mu^{+} \mu^{-}$. subsequent decays take place on time scales $\sim 10^{3} \mathrm{fm} / c$.

We take the final populations of the $\Upsilon(n S)$ states in $p p$ from the CMS data [12] and consider that $27.1 \%$ and $10.5 \%$ of the $\Upsilon(1 S)$ population comes from $\chi_{b}(1 P)$ and $\chi_{b}(2 P)$ decays, respectively [30].

The initial populations are then obtained through an inverted cascade calculation for $p p$ as discussed in [31, 13], and the final suppression factors $R_{A A}(n S)$ in $\mathrm{PbPb}$ for the $\Upsilon(n S)$ states are obtained by applying the feed-down cascade calculation to the preliminary suppression factors. 


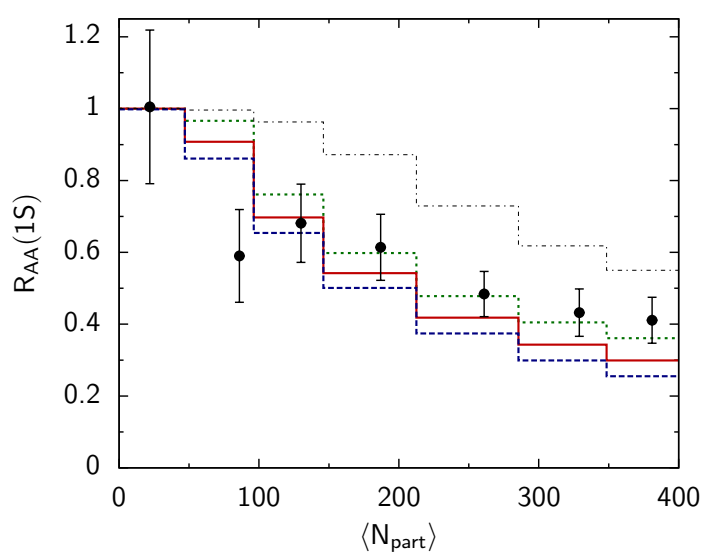

Figure 3: (Color online) Suppression factor $R_{A A}$ for the $\Upsilon(1 S)$ ground state calculated for $2.76 \mathrm{TeV} \mathrm{PbPb}$ collisions from screening, collisional damping, gluodissociation and reduced feed-down using three QGP lifetimes $t_{Q G P}=4,6,8 \mathrm{fm} / \mathrm{c}$ (dotted, solid and dashed line respectively) for the centrality bins $50-100 \%, 40$ $50 \%, 30-40 \%, 20-30 \%, 10-20 \%, 5-10 \%, 0-5 \%$. The dash-dotted upper line is the preliminary suppression factor $R_{A A}^{p r e l}(1 \mathrm{~S})\left(t_{Q G P}=6 \mathrm{fm} / c\right)$ without reduced feed-down. The corresponding CMS data [12] are in good agreement with the model results for the $\Upsilon(1 S)$ state. From [13].

\section{Results}

We present the results for screening and collisional damping derived from the solutions of the Schrödinger equation with the potential eq. (2.1), and the widths for gluodissociation as derived from eq. (3.2). The total decay widths $\Gamma_{t o t}$ are then inserted into a dynamic calculation for the fireball evolution to calculate preliminary suppression factors, eq. (4.1). Subsequently, the bottomium states pass through the decay cascade, to generate the final suppression factors.

Our results for the suppression of the $\Upsilon(1 S)$ state in $\mathrm{PbPb}$ relative to $p p$ are shown in Fig. 3 for three different QGP lifetimes $t_{Q G P}=4,6,8 \mathrm{fm} / c$ as functions of centrality (number of participants). When comparing with our result from the preliminary suppression factor (upper dotted step function), it is evident that the consideration of the feed-down cascade is essential for modeling the suppression. The calculated suppression is in very good agreement with the CMS data for the $\Upsilon(1 S)$ ground state. This is also true for minimum bias (centrality integrated) results, which are discussed in our poster contribution to this conference. The suppression found experimentally for the $\Upsilon(2 S)$ state is, however, much more pronounced than in the calculation [13], in particular, for the three more peripheral data points.

It appears to be very difficult for theoretical models to obtain such a huge suppression of the $\Upsilon(2 S)$ state in peripheral collisions, and indeed, other approaches such as [32, 33, 34] also find that the $\Upsilon(2 S)$ suppression factor rises towards 1 for peripheral collisions. As a consequence of the disagreement with the centrality-dependent data, our minimum-bias results also disagree substantially for the $\Upsilon(2 S)$ and $\Upsilon(3 S)$ states.

The reason for the disagreement will probably be cleared up once more precise $p p$ reference data at $2.76 \mathrm{TeV}$ become available in the future. It is, however, also conceivable that additional suppression mechanisms not considered in this work play a role for the $\Upsilon(2 S)$ and $\Upsilon(3 S)$ states. 


\section{Conclusion}

We have formulated a three-step model for the suppression of the bottomium states $\Upsilon(n S)$ in the quark-gluon plasma that is formed in $\mathrm{PbPb}$ collisions at LHC energies. Due to its stability against screening up to very high temperatures, the $\Upsilon(1 S)$ state is a particularly suitable probe for the relevance of gluodissociation, collisional damping, and reduced feed-down.

We find that gluodissociation of the $\Upsilon(1 S)$ state is sizeable [14] due to the strong overlap of the $\Upsilon(1 S)$ gluodissociation cross section with the thermal gluon distribution. In the temperature region 200-400 MeV, both gluodissociation and collisional damping are found to be important.

The observed suppression factor $R_{A A}(1 S)=0.56$ in minimum-bias $\mathrm{PbPb}$ collisions [12] is essentially due to gluodissociation and damping of the $\Upsilon(1 S)$ state, and to the melting and dissociation of the excited states: The excited states - in particular, the $\chi_{b}(n P)$ states - partially feed the $\Upsilon(1 S)$ state in $p p, p \bar{p}$ and $e^{+} e^{-}$collisions, and their melting and dissociation in the quark-gluon plasma substantially reduces the feed-down in $\mathrm{PbPb}$ collisions at LHC energies.

The calculated $\Upsilon(1 S)$ suppression factor as function of the collision centrality is indeed in very good agreement with the CMS data if the modification of the feed-down cascade in $\mathrm{PbPb}$ as compared to $p p$ is taken into account.

Different from the $\Upsilon(1 S)$ ground state, the excited states - and in particular, the $\Upsilon(2 S)$ and $\Upsilon(3 S)$ states that are observed in the CMS experiment - are already suppressed through screening to a much larger extent than the ground state, so that the contributions from damping and gluodissociation are less important here. The dissolution of the excited states in the quark-gluon plasma causes the substantial feed-down reduction that is one of the three main reasons for the ground-state suppression.

From our calculations it appears that there may be additional causes for the suppression of the excited states, such as cold nuclear matter (CNM) effects - although these should essentially cancel out in the double ratios. It is conceivable that CNM-effects will be constrained in forthcoming $p \mathrm{~Pb}$ measurements at the LHC. Compared to the present CMS experimental results for the suppression of the $\Upsilon(2 S)$ and $\Upsilon(3 S)$ states in $\mathrm{PbPb}$ [12], our calculated $R_{A A}$ values are substantially too large, in particular, in peripheral collisions.

Apart from these detailed deficiencies, it appears that the overall good description of Upsilon suppression in $\mathrm{PbPb}$ collisions at LHC energies in the present formulation contributes to the available indirect evidences for quark-gluon plasma formation in heavy-ion collisions at high relativistic energies.

\section{Acknowledgments}

This work has been supported by the IMPRS-PTFS and the ExtreMe Matter Institute EMMI. A more complete account has been submitted to Phys. Rev. C [13].

\section{References}

[1] T. Matsui and H. Satz, Phys. Lett. B 178, 416 (1986).

[2] B. K. Patra and D. K. Srivastava, Phys. Lett. B 505, 113 (2001). 
[3] L. Kluberg and H. Satz, Landolt Börnstein 23, 373 (2010).

[4] D. E. Kharzeev, J. Phys. G: Nucl. Part. Phys. 34, S445 (2007).

[5] E. T. Atomssa et al., Eur. Phys. J. C 61, 683 (2009).

[6] G. Martinez Garcia et al., J. Phys. G: Nucl. Part. Phys. 38, 124034 (2011).

[7] C. Silvestre et al., J. Phys. G: Nucl. Part. Phys. 38, 124033 (2011).

[8] C.-Y. Wong, 72, 034906 (2005).

[9] H. Masui et al., J. Phys. G: Nucl. Part. Phys. 38, 124002 (2011).

[10] S. Chatrchyan et al., Phys. Rev. Lett. 107, 052302 (2011).

[11] S. Chatrchyan et al., JHEP 1205, 063 (2012).

[12] S. Chatrchyan et al., Phys. Rev. Lett. 109, 222301 (2012).

[13] F. Nendzig and G. Wolschin, submitted to Phys. Rev. C; arXiv:1210.8366 (2012).

[14] F. Brezinski and G. Wolschin, Phys. Lett. B 707, 534 (2012).

[15] Y. Mehtar-Tani and G. Wolschin, Phys. Rev. Lett. 102, 182301 (2009).

[16] W. E. Caswell and G. P. Lepage, Phys. Lett. B 167, 437 (1986).

[17] A. Pineda and J. Soto, Nucl. Phys. B (Proc. Suppl.) 64, 428 (1998).

[18] N. Brambilla, A. Pineda, J. Soto, and A. Vairo, Nucl. Phys. B 566, 275 (2000).

[19] N. Brambilla, J. Ghiglieri, A. Vairo, and P. Petreczky, Phys. Rev. D 78, 014017 (2008).

[20] N. Brambilla et al., JHEP 1009, 038 (2010).

[21] N. Brambilla, M. A. Escobedo, J. Ghiglieri, and A. Vairo, JHEP 1112, 116 (2011).

[22] J. Ghiglieri, arXiv:1201.2920 (2012).

[23] M. Laine, O. Philipsen, M. Tassler, and P. Romatschke, JHEP 0703, 054 (2007).

[24] A. Beraudo, J. P. Blaizot, and C. Ratti, Nucl. Phys. A 806, 312 (2008).

[25] F. Karsch, M. T. Mehr, and H. Satz, Z. Phys. C 37, 617 (1988).

[26] G. Bhanot and M. E. Peskin, Nucl. Phys. B 156, 391 (1979).

[27] F. Nendzig, Ph.D. thesis, University of Heidelberg, in preparation.

[28] H. de Vries, C. W. de Jager, and C. de Vries, Atom. Data Nucl. Data Tabl. 36, 495 (1987).

[29] K. Yagi, T. Hatsuda, and Y. Miake, Quark-Gluon Plasma, pp. 245-277 (Cambridge University Press, Cambridge, UK, 2008).

[30] T. Affolder et al., Phys. Rev. Lett. 84, 2094 (2000).

[31] F. Brezinski and G. Wolschin, Phys. Lett. B 707, 534 (2012).

[32] T. Song, K. C. Han, and C. M. Ko, Phys. Rev. C 85, 014902 (2012).

[33] A. Emerick, X. Zhao, and R. Rapp, Eur. Phys. J. A 48, 72 (2012).

[34] M. Strickland and D. Bazow, Nucl. Phys. A 879, 25 (2012). 